\section{Microscopy Coming Events}

2016

Physics and Chemistry of Semiconductor Surfaces and Interfaces (PCSI-43) January $17-21,2016$

Location: Palm Springs, CA

www.pcsiconference.org

24th Australian Conference on Microscopy and Microanalysis January 31-February 4, 2016

Melbourne, Australia

www.acmm2016.org

Nanoscience and Nanotechnology (ICONN) 2016

February 7-11, 2016

Canberra, Australia

www.ausnano.net/iconn2016

60th Annual Meeting, Biophysical Society

February 27-March 2, 2016

Los Angeles, CA

www.biophysics.org/Meetings/AnnualMeeting/ tabid/85/Default.aspx

PITTCON Conference

March 6-10, 2016

Atlanta, GA

http://pittcon.org

2016 MRS Spring Meeting

March 28-April 1, 2016

Phoenix, AZ

www.mrs.org/spring2016

Microscopy \& Microanalysis 2016

July 24-28, 2016

Columbus, $\mathrm{OH}$

www.microscopy.org

European Microscopy Congress

August 28-September 2, 2016

Lyon, France

http://emc2016.fr

\section{7}

Microscopy \& Microanalysis 2017

July 23-27, 2017

St. Louis, MO

www.microscopy.org

\section{8}

Microscopy \& Microanalysis 2018

August 5-9, 2018

Baltimore, MD

www.microscopy.org

2019

Microscopy \& Microanalysis 2019

August 4-8, 2019

Portland, OR

www.microscopy.org

\section{0}

Microscopy \& Microanalysis 2020 August 2-6, 2020

Milwaukee, W

www.microscopy.org

More Meetings and Courses Check the complete calendar near the back of this magazine.

\title{
More Views Give Better Spatial and Temporal Resolution of Whole Organisms
}

\author{
Stephen W. Carmichael \\ Mayo Clinic, Rochester, MN 55905 \\ carmichael.stephen@mayo.edu
}

Light-sheet microscopy methods have recently been developed for highspeed imaging but have limitations, such as limited penetration, that limits specimen volume size. Powerful strategies have been proposed for improving resolution in light-sheet microscopy, but each has its limitations, such as increased photo-damage that restricts observation times of dynamic events. More recently Raghav Chhetri, Fernando Amat, Yinan Wan, Burkhard Höckendorf, William Lemon, and Philipp Keller have developed another strategy for resolution enhancement that is based on multiview imaging. Their method provides uniform spatial resolution in all dimensions (isotropy) and is labeled IsoView lightsheet microscopy. IsoView light-sheet microscopy rapidly images large specimens via simultaneous light-sheet illumination and fluorescence detection along four orthogonal directions. Combining these four views by means of high-throughput multiview deconvolution yields images with high resolution in all three dimensions.

The specimen is imaged along different directions, which yields different relative orientations. Whereas two views give good resolution, Chhetri et al. designed a microscope with four orthogonal arms for simultaneous light-sheet illumination and fluorescence detection. This yields a massive number of data points that require correspondingly massive computing power, yet these authors

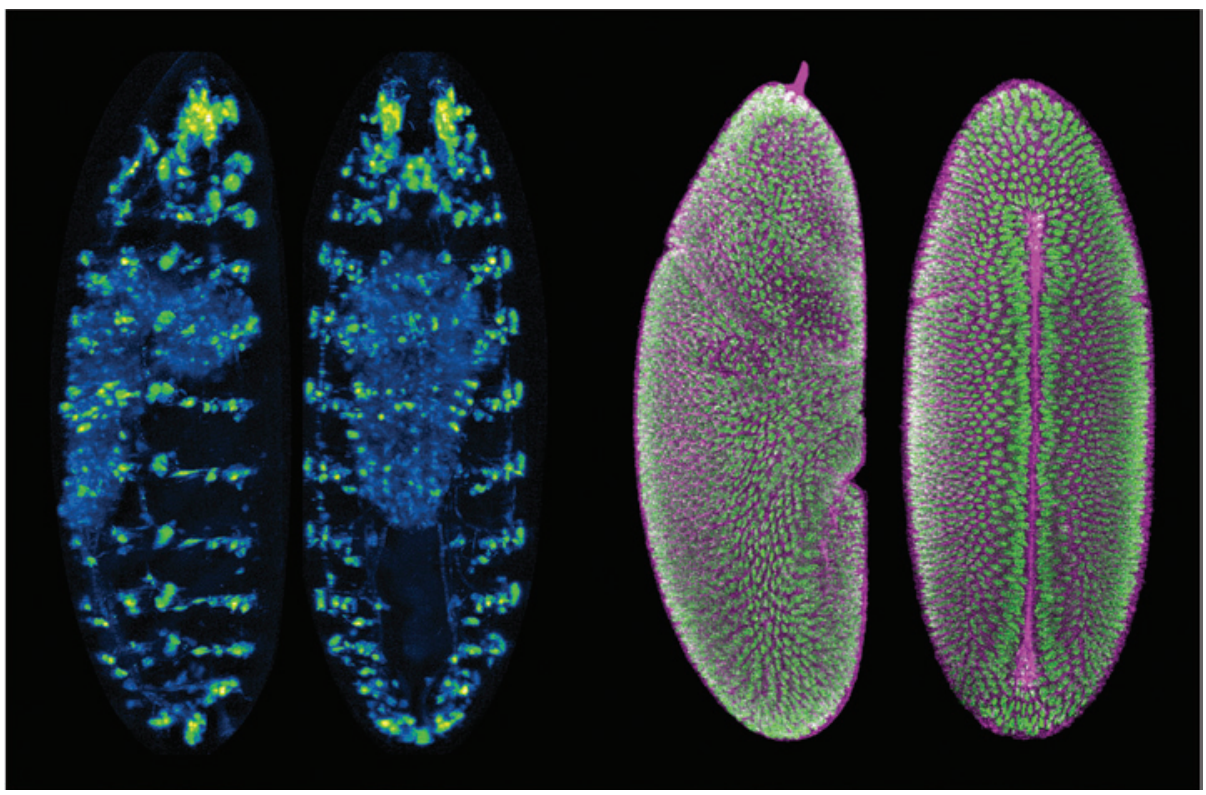

Figure 1: IsoView whole-animal functional images of Drosophila embryos. The length of the embryos is approximately $500 \mu \mathrm{m}$. The two images on the left are maximum-intensity projections of deconvoluted image data of a stage 17 embryo expressing the calcium indicator GCaMP6s throughout the nervous system. The two images on the right are four-view two-color images of gastrulating embryos with labeled nuclei (His2Av-mRFP1) and membranes (Spider-GFP) using a combination of two IsoView modes. 


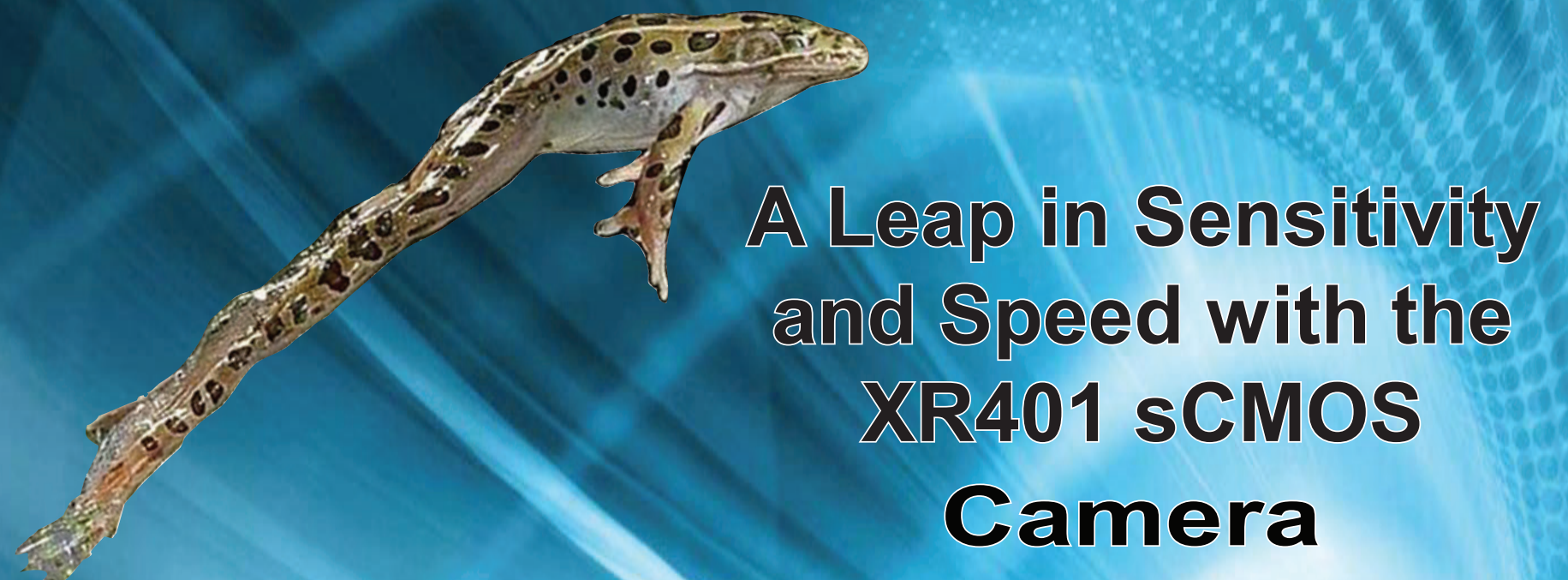

- Cryo TEM

- Low Dose TEM

- Diffraction

- In-Situ TEM

- Extraordinary speed

- Non-Blooming Sensor $0<2$ e/pixel noise

Adenovirus

Dr. Cameron Ackerley

The Hospital for Sick Children

\section{A Leap in Sensitivity and Speed with the XR401 sCMOS Camera}
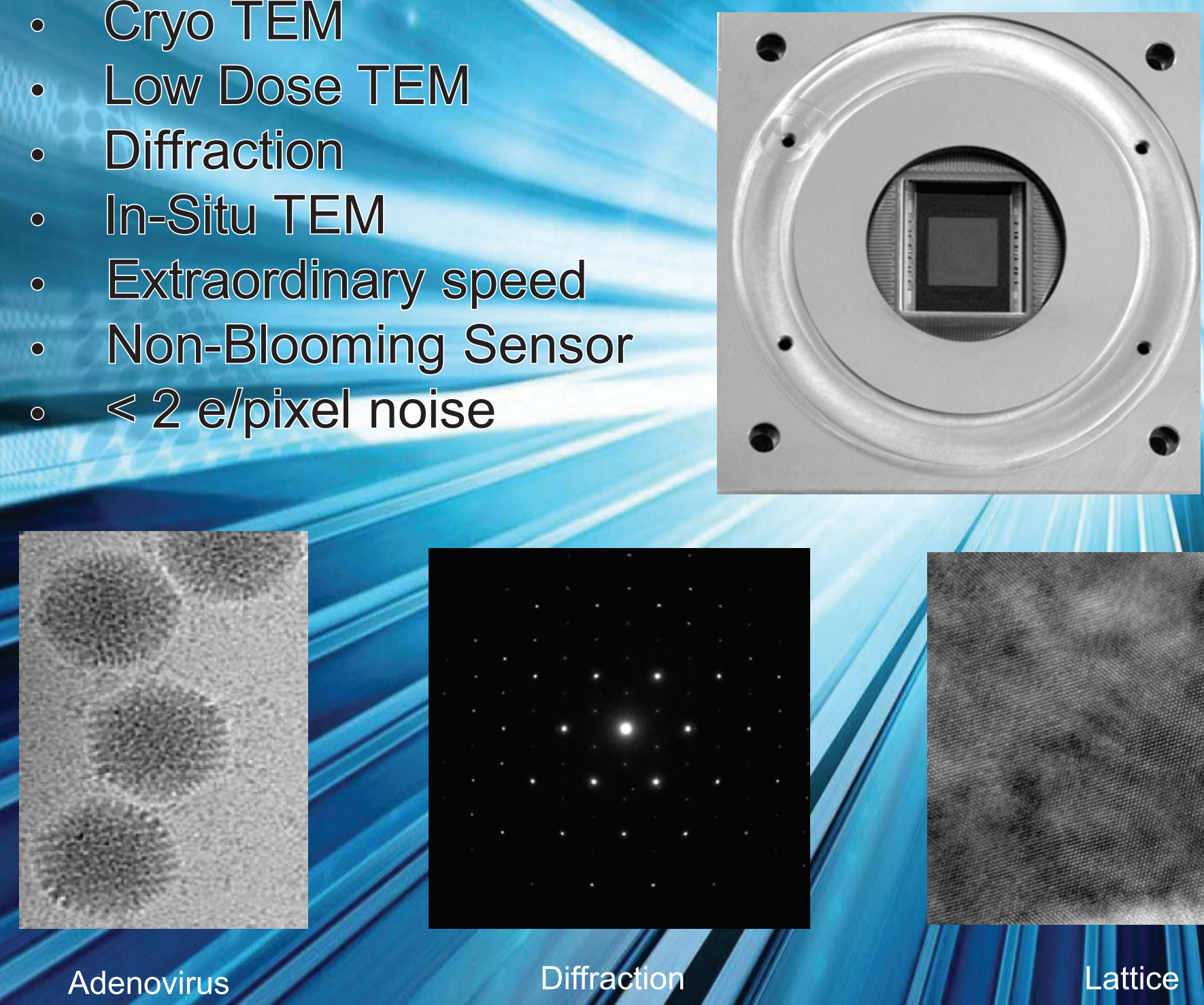

Dr. Pengfei Hu at the Shanghai University

Lattice 
developed algorithms that decreased the computational time from months to about two days. They also designed customized objectives that provide long working distances and high numerical apertures. They provide specifics on the design of these objectives as well as detailed blueprints for the IsoView microscope.

Using this elegant instrument, Chhetri et al. demonstrated whole-animal functional imaging of fruit fly (Drosophila) embryos at a spatial resolution of about $2 \mu \mathrm{m}$ and a temporal resolution of $2 \mathrm{~Hz}$ for several hours. They also obtained spatially isotropic whole-brain functional imaging in zebrafish (Danio rerio) larvae and spatially isotropic multicolor imaging of rapid cellular dynamics across fruit fly embryos.

Compared to conventional light-sheet microscopy, IsoView microscopy improves three-dimensional spatial resolution and decreases resolution anisotropy. For Drosophila embryos, scattering and aberrations are relatively strong, but resolution varies with depth. The authors performed a systematic depth-dependent analysis of the resolution improvement achieved by IsoView in this scenario (in Supplementary Figure 7 in [1]). Conventional resolution is approximately $1.8 \mu \mathrm{m}$ laterally and $5.5 \mu \mathrm{m}$ axially at a depth of 20 to $30 \mu \mathrm{m}$, whereas IsoView provides 1.1 to $1.8 \mu \mathrm{m}$ laterally and axially. At maximum imaging depth, conventional resolution is approximately $3.0 \mu \mathrm{m}$ laterally and $9.0 \mu \mathrm{m}$ axially, where IsoView provides 1.3 to $2.5 \mu \mathrm{m}$ laterally and axially.

Compared with existing high-resolution light-sheet techniques, IsoView microscopy effectively doubles the penetration depth and provides sub-second temporal resolution for specimens 400 -fold larger than could previously be imaged. The functional imaging capabilities of this elegant microscope allows for the first time the ability to capture neuronal activity simultaneously throughout the entire nervous system of an intact living organism with more than 10,000 neurons. By contrast, the largest organisms that could previously be fully covered were C. elegans, which have a relatively simple nervous system with only a few hundred neurons. This is a remarkable achievement that provides enormous power to observe dynamic cellular events in whole organisms!

\section{References}

[1] RK Chhetri et al., Nat Methods, doi:101038/NMETH.3632, 2015.

[2] The author gratefully acknowledges Drs. Philipp Keller and Raghav Chhetri for reviewing this article, as well as Dr. Charles Lyman for helpful editing.
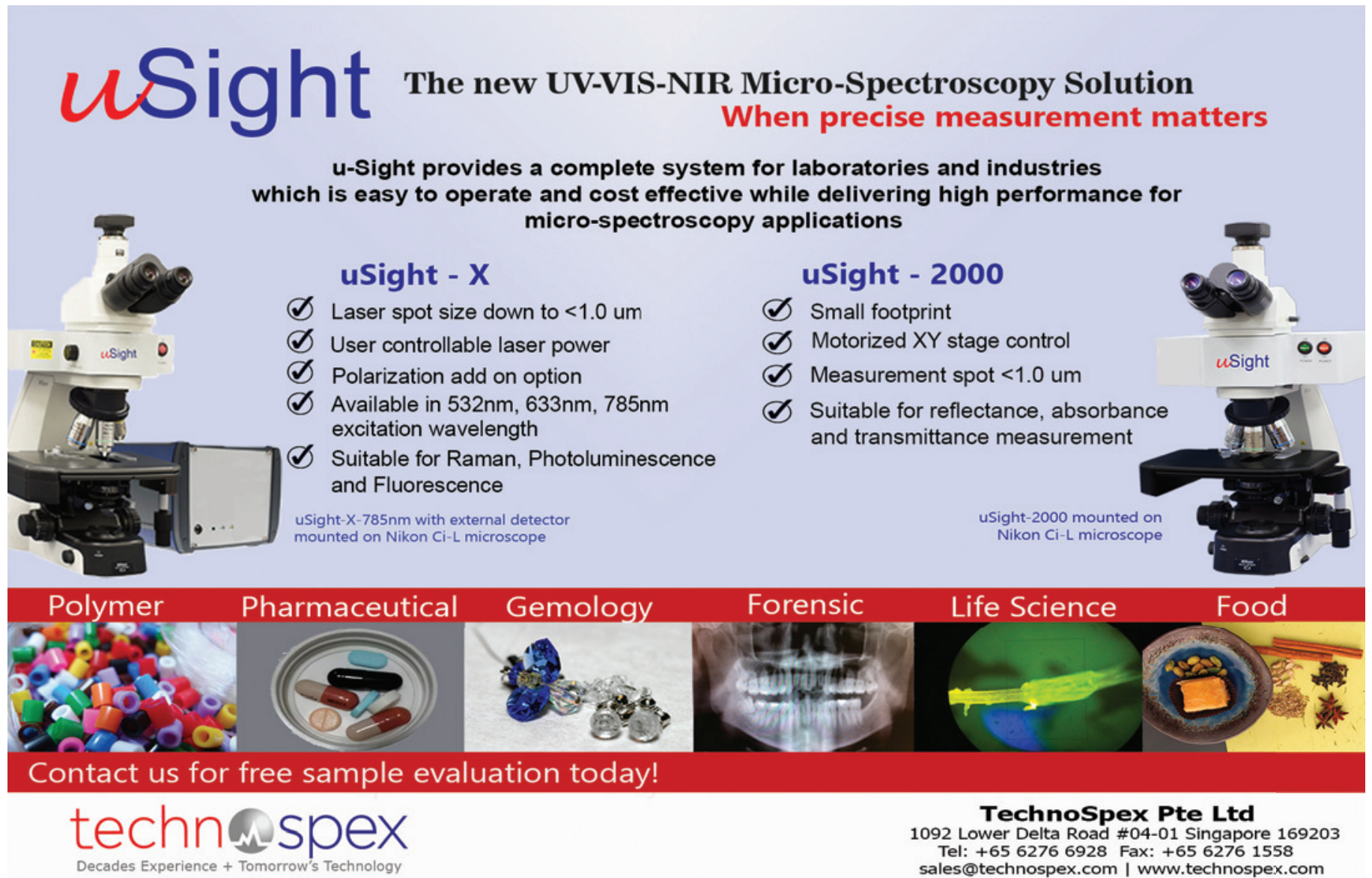

TechnoSpex Pte Ltd 1092 Lower Delta Road \#04-01 Singapore 169203 Tel: +65 62766928 Fax: +65 62761558 sales@technospex.com | www.technospex.com 


\section{Powerful LED Illumination for All Your Fluors}

X-Cite ${ }^{\circledR}$ TURBO with LaserLED Hybrid Drive ${ }^{\text {TM }}$ for fluorescence microscopy

High power, multi-wavelength LED illumination

Fast-switching LEDs with exceptional uniformity and stability

Simple to advanced LED control

Contact X-Cite today to learn more!

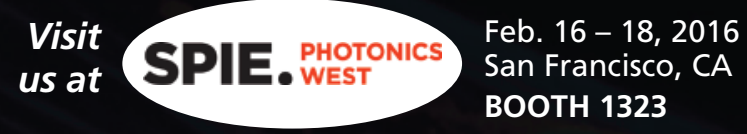

